\title{
FAST MAGNETIC RECONNECTION VIA JETS AND CURRENT MICROSHEETS
}

\author{
P. G. WATSON \\ Center for Magnetic Reconnection Studies, Institute for Fusion Studies, Department of Physics, University of Texas at Austin, \\ 1 University Station C1600, Austin, Texas 78712; pgwatson@kato.ph.utexas.edu \\ AND \\ I. J. D. CRAIG \\ Department of Mathematics, University of Waikato, Private Bag 3105, Hamilton, New Zealand; math0097@waikato.ac.nz \\ Received 2003 March 28; accepted 2003 April 29; published 2003 May 7
}

\begin{abstract}
Numerical simulations of highly nonlinear magnetic reconnection provide evidence of ultrathin current microsheets. These small-scale sheets are formed by strong exhaust jets from a primary large-scale current layer. The overall size of the secondary microsheet is determined by the thickness of the primary sheet. Preliminary scalings show that the thickness of the microsheet varies linearly with the plasma resistivity. This scaling suggests that microsheets may provide fast reconnection sites in magnetically complex plasmas such as the solar corona and planetary magnetospheres.
\end{abstract}

Subject headings: galaxies: jets — MHD — plasmas — Sun: flares — Sun: X-rays, gamma rays

\section{INTRODUCTION}

Magnetic reconnection is believed to play an important role in a variety of explosive energy-release mechanisms observed in astrophysical and laboratory plasmas, for example, solar flares, geomagnetic substorms, and tokamak disruptions. In most studies of magnetic reconnection, the merging is powered by a large-scale velocity field that washes magnetic plasma toward a localized diffusion region (see Priest \& Forbes 2000). A critical feature of the mechanism is the collapse of the magnetic field to small length-scales. This collapse is halted by the plasma resistivity $\eta$, and although $\eta$ is very small for typical astrophysical plasmas, it is thought to determine both the speed and strength of the magnetic energy release. Accordingly, reconnection theory has focused on the dependence of the merging rate on the plasma resistivity. It is commonly believed that the energy release in many space and laboratory plasmas requires a "fast" reconnection mechanism in which the reconnection rate is independent of resistivity.

The purpose of this Letter is to point out that the exhaust region of a large-scale current layer can provide the external source for small-scale secondary reconnection events. Numerical simulations show that ejecta from the primary sheet act rather like a turbo mechanism, enhancing dissipation by supplying high-pressure collimated jets to sustain the microsheet. Although the primary sheet has an overall length comparable to the size of the computational domain, the microsheet is very much smaller, its length being controlled by the narrow exhaust jet of the primary current layer. Such microsheets have the potential to act as extremely short-lived localized sites of energy release (e.g., X-ray-bright points) in magnetically complex plasmas such as the solar corona.

The exhaust jets themselves are also of some interest. In our simulations, the jets emerge from an initially global flow via the action of the magnetic field. The mechanism involves the formation of a magnetic slingshot that accelerates fluid from the end of a reconnecting current sheet into a strongly collimated flow. This mechanism could have an important role to play in the formation of astrophysical jets observed in active galactic nuclei and protostellar objects, and it contrasts somewhat with other proposed MHD acceleration scenarios (e.g.,
Blandford \& Payne 1982), where the magnetic slingshot effect is generated by rotation.

In $\S 2$, we describe planar simulations that lead to the formation of current microsheets. We also discuss the scalings with resistivity of the primary and secondary current layers. Section 3 summarizes our findings.

\section{THE RECONNECTION SIMULATION}

\subsection{The Numerical Experiment}

We use the planar incompressible code of Craig \& Watson (1999), which includes the effects of resistivity $\eta$ and viscosity $\nu$, to perform the numerical experiments on the doubly periodic domain $(-1,1) \times(-1,1)$. The code employs stream and flux function representations for the velocity and magnetic fields, $\boldsymbol{v}(x, y, t)=\boldsymbol{\nabla} \phi \times \hat{z}, \boldsymbol{B}(x, y, t)=\boldsymbol{\nabla} \psi \times \hat{z}$, and has been checked by Heerikhuisen, Craig, \& Watson (2000) and Watson \& Craig (2001) against a family of analytic reconnection models (Craig \& Henton 1995; Craig \& Fabling 1996; Craig \& Watson 2000). The present results, obtained using a fourth-order accurate version of the program, have also been reproduced using a spectral code and the compressible FLASH code (Fryxell et al. 2000).

Simulations involving microsheets begin with the initial condition

$$
\begin{aligned}
& \phi=\alpha \sin (\pi x) \sin (\pi y) / \pi, \quad \alpha>0, \\
& \psi=\epsilon \cos (\pi x) / \pi .
\end{aligned}
$$

This system comprises four counterrotating flow cells and an initially one-dimensional vertical magnetic field. Given the periodic boundary conditions and the nature of the flow, a twodimensional field structure soon develops and magnetic reconnection ensues at a periodic array of $X$-type magnetic nulls located at $(x, y)=(0, \pm 1),(x, y)=( \pm 1,0)$. The reconnection proceeds via an initial implosion phase, during which the magnetic field is swept toward the nulls and amplified forming quasi-one-dimensional current sheets. 

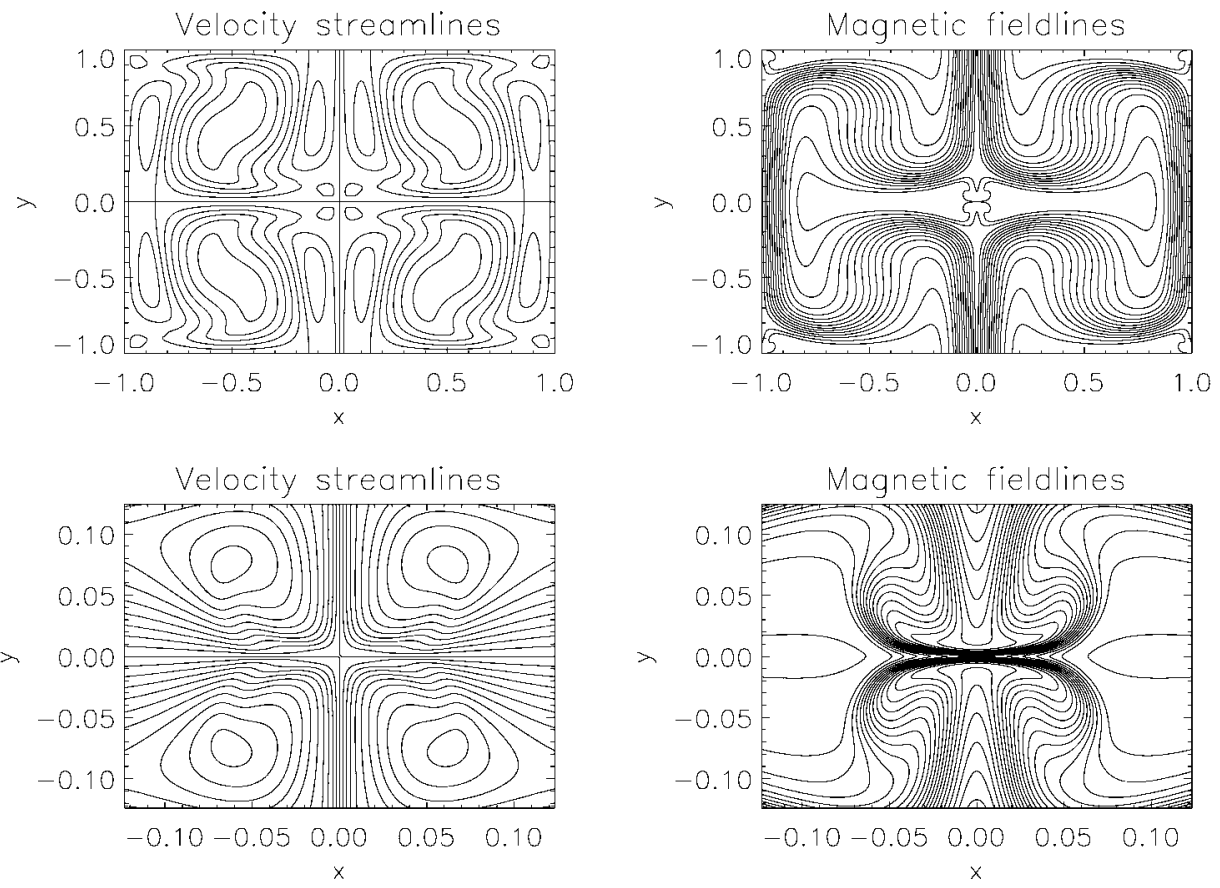

FIG. 1.-Velocity streamlines and fieldlines at the time of maximum current in the microsheet. The top row of plots shows the flow and field over the entire domain, while the bottom row shows a blow-up of these same profiles in the vicinity of the microsheet located at the origin.

\subsection{The Primary and Secondary Current Sheets}

The properties of the primary current sheet follow the theory described by Craig \& Watson (1999). There are two regimes to consider. In the presaturation regime, the magnetic pressure in the sheet is relatively weak, and so it can be intensified by systematically reducing the plasma resistivity $\eta$. In this phase, the reconnection rate is fast (Sonnerup \& Priest 1975; Craig \& Henton 1995) and the properties of the solution scale in accordance with analytic predictions (Craig \& Watson 1999). A value of $\eta$ is reached, however, when the back pressure of the field in the current sheet is sufficient to stall the background flow. The sheet "saturates," and the solution appears to enter a slow reconnection phase, superficially resembling SweetParker. In reality the saturated regime is very complicated, and

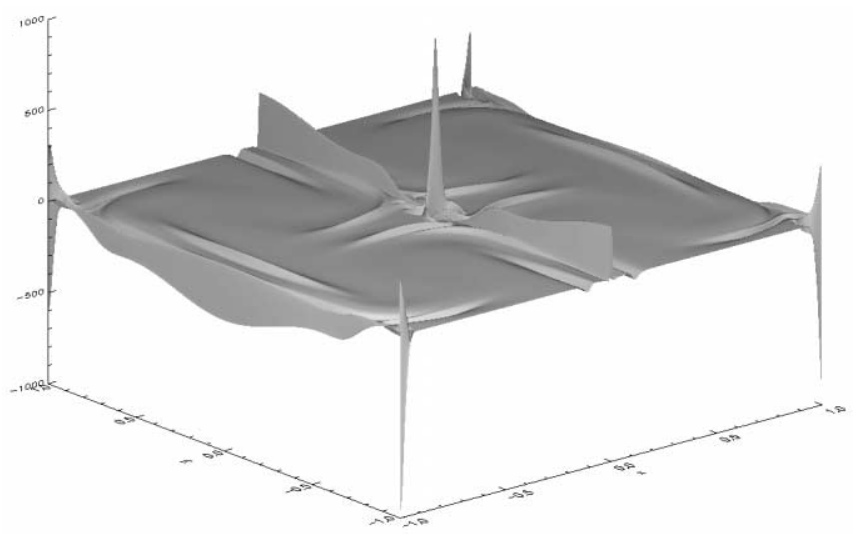

Fig. 2.-Surface plot of the current of the run shown in Fig. 1. The primary sheet is still evident, running down the middle of the domain (and along the edges of the box). The secondary microsheet appears as a sharp spike at the center of the box (and at the four corners). there is some evidence for the emergence of disparate spatial scales and temporal chaos (Craig \& Watson 1999).

It is the exhaust of reconnected flux from the primaries that powers the secondary microsheets. The ejected horizontal field, advected by strong exhaust jets, generates secondary sheets at $(x, y)=(0,0)$ and at the four corners of the box. In the presaturation regime, the secondary sheets resemble scaled down versions of the primaries. The secondary sheets convert the horizontal field back into vertical field and in doing so generate tertiary current layers at the location of the old primaries. The whole process then repeats itself and reconnection proceeds in a regular periodic cycle, with energy and flux being liberated at each reconnection event.

This picture changes dramatically once the primaries enter the saturation phase. The secondary sheets now change their character in response to the increasingly narrow collimated jets that emerge from the primary, becoming current "microsheets." The periodic nature of the reconnection also disappears, and the temporal behavior becomes increasingly chaotic.

A typical simulation is shown in Figure 1. The parameter values for this run are $\alpha=1, \epsilon=0.3$, and $\eta=\nu=0.0001$. The top two plots show the flow streamlines and magnetic fieldlines over the entire box at the time of maximum current in the secondary. In these plots, the primary sheets are still clearly visible as accumulations of field around the nulls at the top, bottom, and sides of the domain. That saturation has occurred is evidenced by the fact that the flow has stagnated in the vicinity of the primary sheets. The small secondary sheet is just visible at the center of the domain in the magnetic field plot. The bottom two plots show close-ups of the flow and field in the vicinity of the microsheet. Of particular note here are the strong collimated vertical jets evident in the flow profile. Figure 2 shows the corresponding surface plot of the current density. While the large-scale primary sheets are still visible, 


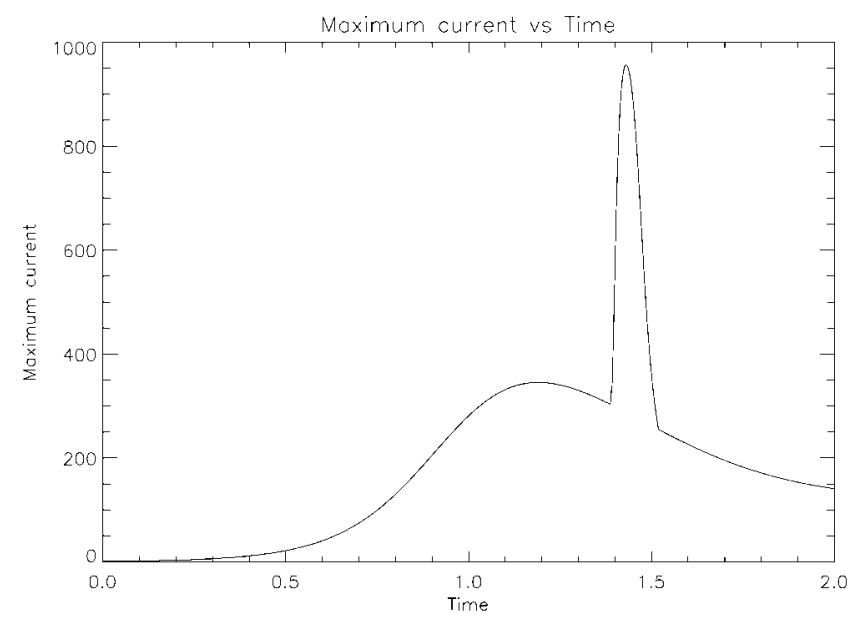

FIG. 3.-Plot of the maximum current vs. time. The current in the primary peaks around $t=1.2$, while the sharp feature near $t=1.4$ relates to the extremely intense and short-lived microsheet.

they are overshadowed by the sharp, almost spikelike current features associated with the microsheets.

Figure 3 shows the evolution of the maximum current over the domain with time. The initial implosion, which occurs over a timescale of 1.2 Alfvén times, results in the primary sheet, while a second, much faster localization results in the strong secondary current spike near $t=1.4$. The timescales involved in the development and lifetime of the secondary sheet are much shorter than those of the primary.
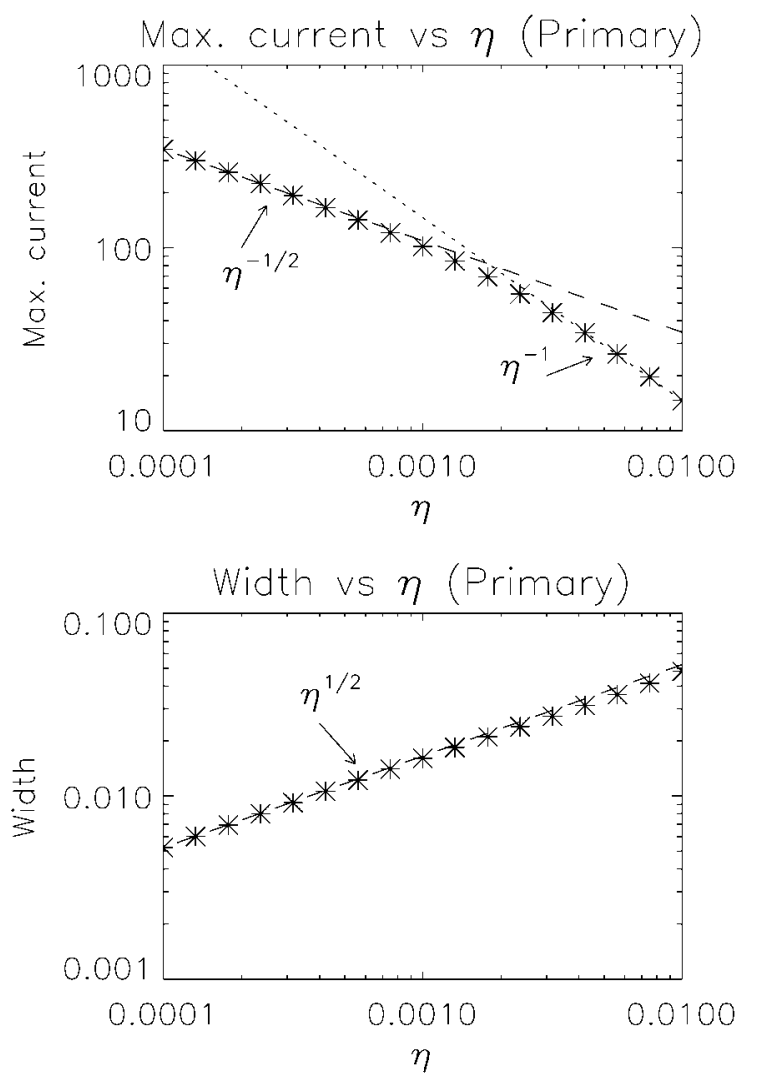

\subsection{Resistive Scalings}

In Figure 4, we show key diagnostics for the primary and secondary sheets. The top row of diagrams shows how the maximum current in the two sheets scales with resistivity. The first plot (for the primary) can be explained by the scaling arguments presented in Craig \& Watson (1999). In the presaturation regime $(\eta \geq 0.002)$, the reconnection is fast and the maximum current scales as $\eta^{-1}$. Once the field in the sheet saturates $(\eta \lesssim 0.002)$, the reconnection slows and the solution enters a slow SweetParker regime in which the maximum current scales as $\eta^{-1 / 2}$. The maximum current in the secondary sheet (Fig. 4, top right) also undergoes changes when the primary sheet saturates. Of particular note is the fact that after saturation, the maximum current in the secondary grows significantly faster than $\eta^{-1}$.

The bottom row shows the scaling of the primary and secondary sheet widths with $\eta$. The width of the primary sheet (Fig. 4, bottom left) scales as $\eta^{1 / 2}$ in the presaturation phase, as expected. It is a remarkable fact that this scaling carries over into the saturation regime. In contrast, the secondary sheet undergoes a change near $\eta \simeq 0.002$, where the primary sheet saturates. After saturation, the width appears to obey an $\eta^{1}$ scaling law, indicating that the secondary sheet is very much thinner than the primary.

The scalings of the secondary sheet are enticing. In the saturation regime (of the primary), the reconnection rate $E=$ $\eta J$, where $J$ is the current density at the null, is superfast (i.e., it scales as a weak inverse power of $\eta$ ). Since the sheet field and the exhaust velocity in the secondary are still increasing with reductions in $\eta$, we cannot expect this behavior to continue
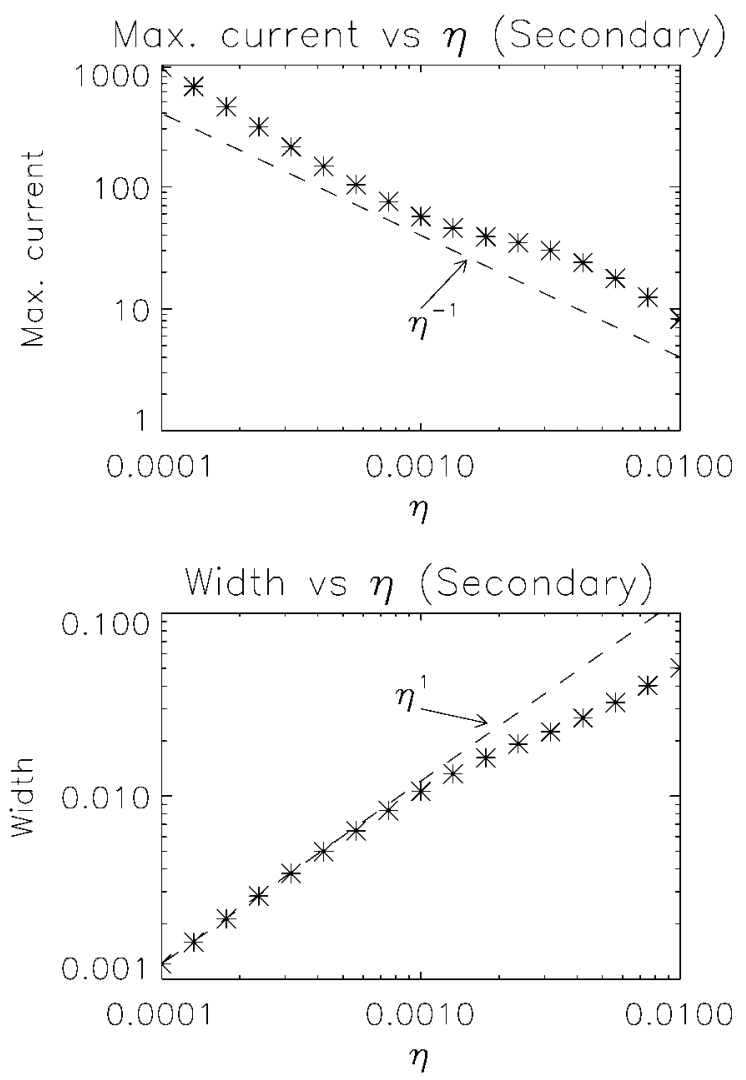

FIG. 4.- Scalings of various sheet characteristics vs. resistivity for both the primary (left) and secondary (right) sheets. The top row of diagrams shows the scaling of the maximum current in the sheet with $\eta$. The bottom row shows the scaling of the sheet widths. 
indefinitely. However, given that the width of the secondary sheet scales as $\eta$, the current can still behave as $\eta^{-1}$ even after saturation of the secondary field. The implication is that the reconnection rate will eventually switch from superfast to fast (i.e., $\eta J$ will become independent of $\eta$ ). The reconnection rate in the microsheet is then fast for arbitrarily small values of the plasma resistivity.

These scalings are reminiscent of Petschek's reconnection mechanism (Petschek 1964). Although there may be some evidence that MHD shocks are forming (see the blow-up regions in Fig. 1), the microsheets display several differences to the simple Petschek model: the angle of the field separatrices is very small; the sheet aspect ratio increases with reductions in resistivity (there are indications that the aspect ratio is becoming fixed, but we have not been able to investigate small enough values of $\eta$ to confirm this); and there is no appreciable accumulation of current along the field separatrices. However, it is possible that we are observing the first stages of a transition from Sweet-Parker to Petschek reconnection.

Finally, we should emphasize that the results presented here are robust to the addition of small-scale turbulent disturbances and insensitive to whether the reconnection is head-on (as described in this Letter) or appreciably sheared. This robustness to the breaking of the fourfold symmetry of the simulations presented here suggests that microsheets may occur in quite general settings. Indeed, here we have relied on the collision of two jets to generate a microsheet, but it seems likely that even the highly asymmetric configuration of one jet colliding with a stationary magnetic field might give rise to a microsheet. The emergence of microsheets, however, is sensitive to tuning the relative magnitudes of the initial flow and field strengths $\alpha$ and $\epsilon$.

\section{DISCUSSION AND CONCLUSIONS}

The simulations presented here suggest that magnetically complex regions such as the corona and the magnetosphere may, at least potentially, possess a hierarchy of current-sheet structures. The primary current layers produce narrow Alfvénic exhaust jets that feed the secondary current microsheets and so on. Although the bulk of the energy release probably occurs in the primary structures, microsheets powered by primary ejecta could well account for localized hot spots within the plasma (e.g., X-ray-bright points associated with solar flares). This idea of a hierarchy of small-scale secondary sheet structures is not new. Biskamp (1993), in a series of steady state simulations, also observed the formation of secondary sheets. However, as his secondary sheets are self-similar copies of the main sheet, and given that they are also aligned parallel to it, their generation mechanism must be somewhat different from the current microsheets found in our simulations.

Perhaps the key observation is the very small time and lengthscales associated with the microsheets. The present results imply that transient magnetic reconnection may occur over a small fraction of an Alfvén time in a small-scale sheet whose thickness scales as $\eta^{1}$, characteristic of fast reconnection solutions.

Finally, our results suggest that under certain circumstances, magnetic reconnection events may act as sources of strongly collimated jets. Other MHD jet formation models have been proposed that rely on rotation as a global acceleration mechanism (Blandford \& Payne 1982), but reconnection-driven jets may provide an attractive alternative for small-scale jet generation.

We are grateful to Sean Oughton for confirming these results using a spectral code. This work was supported by the US Department of Energy Contract DE-FC02-01ER-54652 under the auspices of the program for Scientific Discovery through Advanced Computing.

\section{REFERENCES}

Biskamp, D. 1993, Nonlinear Magnetohydrodynamics (Cambridge: Cambridge Univ. Press)

Blandford, R. D., \& Payne, D. G. 1982, MNRAS, 199, 883

Craig, I. J. D., \& Fabling, R. B. 1996, ApJ, 462, 969

Craig, I. J. D., \& Henton, S. M. 1995, ApJ, 450, 280

Craig, I. J. D., \& Watson, P. G. 1999, ApJ, 516, 924 2000, Sol. Phys., 194, 251

Fryxell, B., et al. 2000, ApJS, 131, 273
Heerikhuisen, J., Craig, I. J. D., \& Watson, P. G. 2000, Geophys. Astrophys. Fluid Dyn., 93, 115

Petschek, H. E. 1964, in AAS/NASA Symp. on Physics of Solar Flares, ed. W. N. Hess (NASA SP-50; Washington, DC: NASA), 425

Priest, E. R., \& Forbes, T. G. 2000, Magnetic Reconnection MHD Theory and Applications (Cambridge: Cambridge Univ. Press)

Sonnerup, B. U. Ö., \& Priest, E. R. 1975, J. Plasma Phys., 14, 283

Watson, P. G., \& Craig, I. J. D. 2001, J. Geophys. Res., 106, 15,735 